\title{
Exploring natural gems and minerals by multiphoton microscopy
}

Cromey, Benjamin, Knox, Ryan, Fritz, Eric, Kieu, Khanh

Benjamin Cromey, Ryan J. Knox, Eric Fritz, Khanh Kieu, "Exploring natural gems and minerals by multiphoton microscopy," Proc. SPIE 11099, Light in Nature VII, 1109902 (30 August 2019); doi: 10.1117/12.2528764

SPIE Event: SPIE Optical Engineering + Applications, 2019, San Diego, California, United States 


\title{
Exploring Natural Gems and Minerals by Multiphoton Microscopy
}

\author{
Benjamin Cromey ${ }^{\mathrm{a},}{ }^{*}$, Ryan J. Knox ${ }^{\mathrm{a}}$, Eric Fritz ${ }^{\mathrm{b}}$, and Khanh Kieu ${ }^{\mathrm{a}}$ \\ a James C. Wyant College of Optical Sciences, The University of Arizona, 1630 E University \\ Blvd, Tucson, AZ 85721, USA \\ ${ }^{b}$ Department of Geosciences, The University of Arizona, 1040 E 4th St, Tucson, AZ 85721, \\ USA
}

\begin{abstract}
The beauty of gems and minerals have been examined and appreciated by optical tools for centuries. Current methods for examining the interior structure of gems and minerals typically requires a sample to be cut and polished prior to imaging. In this presentation, we introduce a new tool for imaging gems and minerals in three dimensions, the multiphoton microscope. We have demonstrated that the multiphoton microscope can capture fascinating information from natural gems and minerals with sub-micron resolution at depths up to the millimeter scale. This new application of multiphoton microscopy may open the doors to non-destructive characterization leading to new information on the formation, structure, and appearance of these stones that have fascinated the eye for centuries.
\end{abstract}

Keywords: optics in nature, nonlinear optics, multiphoton microscopy, geosciences, mineralogy

\section{INTRODUCTION}

Gems and minerals have fascinated the casual and scientific observer alike for thousands of years. Recently, many different imaging and analysis approaches have been used to study gems and minerals, including polarized light microscopy, ${ }^{1}$ Raman spectroscopy, ${ }^{2-8}$ Atomic Force Microscopy, ${ }^{9}$ hyperspectral imaging, ${ }^{10}$ Terahertz Spectroscopy ${ }^{11,12}$ Electron Microscopy, ${ }^{13}$ and X-ray micro CT scans, ${ }^{14}$ each with advantages and disadvantages. Most of these methods require samples to be carefully prepared, which usually involves cutting into a stone and polishing a plane of interest. However, a non-destructive method of imaging planes of interest within a bulk sample would be very impactful to a science with such valuable samples, as it would preserve the samples, reduce the time needed to study them, and introduce the possibility of discovering new information.

We have recently published our work demonstrating that a multiphoton microscope can three-dimensionally image gem and mineral samples with high resolution. ${ }^{15}$ Multiphoton microscopes are more traditionally used in the biological sciences, and have been very impactful in high profile studies in identifying diseases without stains. ${ }^{16,17}$ Our group has used our multiphoton both for biological and material studies, proving the versatility of the instrument. ${ }^{18-21}$ The same physics that allows a multiphoton microscope to look three-dimensionally through biological tissue also enables imaging through crystalline structures, and these crystalline structures frequently have very strong signal, which creates beautiful images with a high signal to noise ratio. We are continuing our work in this area through a collaboration with the University of Arizona Geosciences department to further explore the usefulness of this approach to the geological sciences. These images are both useful and beautiful, and as such we are excited to share them with the scientific community.

For this study, a wide variety of stones were examined, including a set of polished and unpolished stones from the set of so-called "Birth Stones." Several samples of each stone were provided to us by Eric Fritz from the University of Arizona Geosciences Department, some which were cut and polished, and others of which were the untouched mineral. In addition to these samples, we re-examined several stones from the previous publication, as well as minerals from the local area. Because this conference is about the beauty of optics and nature, this paper will be more qualitative, and we recommend the reader pursue reading our original publication for a more rigorous introduction to these studies. ${ }^{15}$

\footnotetext{
*bcromey@email.arizona.edu,
} 


\section{MULTIPHOTON MICROSCOPY DESCRIPTION}

A brief description of multiphoton microscopy (MPM) will be given here. Several references explain the physics and designs of the microscope in greater detail for the interested reader. ${ }^{17,18}$ In our system, a pulsed femtosecond fiber laser is raster-scanned using a pair of galvanomateric mirrors, and that scanning is translated to the sample via a relay beam-expanding telescope and a microscope objective. Once focused at the sample, the very high peak power (kilowatt level) laser light interacts nonlinearly with the sample, creating new wavelengths in the process. Because the laser is pulsed, the average power is low, preventing damage to the sample. In the images, we used primarily two effects, second harmonic and third harmonic generation. Second harmonic generation (SHG), where two photons scatter and emit a single photon with both their energies, is generated from regions with broken symmetry, or from non-centrosymmetric crystal structures. Third harmonic generation is created from boundaries between regions of different refractive index, or more precisely different third order nonlinear response $\chi_{3}$, which is a very small contribution to the refractive index. Further explanations on these effects can be found in the book on Nonlinear Optics by Boyd. ${ }^{22}$ In our microscope, we use both a $1040 \mathrm{~nm}$ and $1550 \mathrm{~nm}$ fiber laser as our sources, both of which were used for this project.

\section{IMAGE RESULTS}

A subset of images from the birthstone samples can be seen in Fig. 1 below.
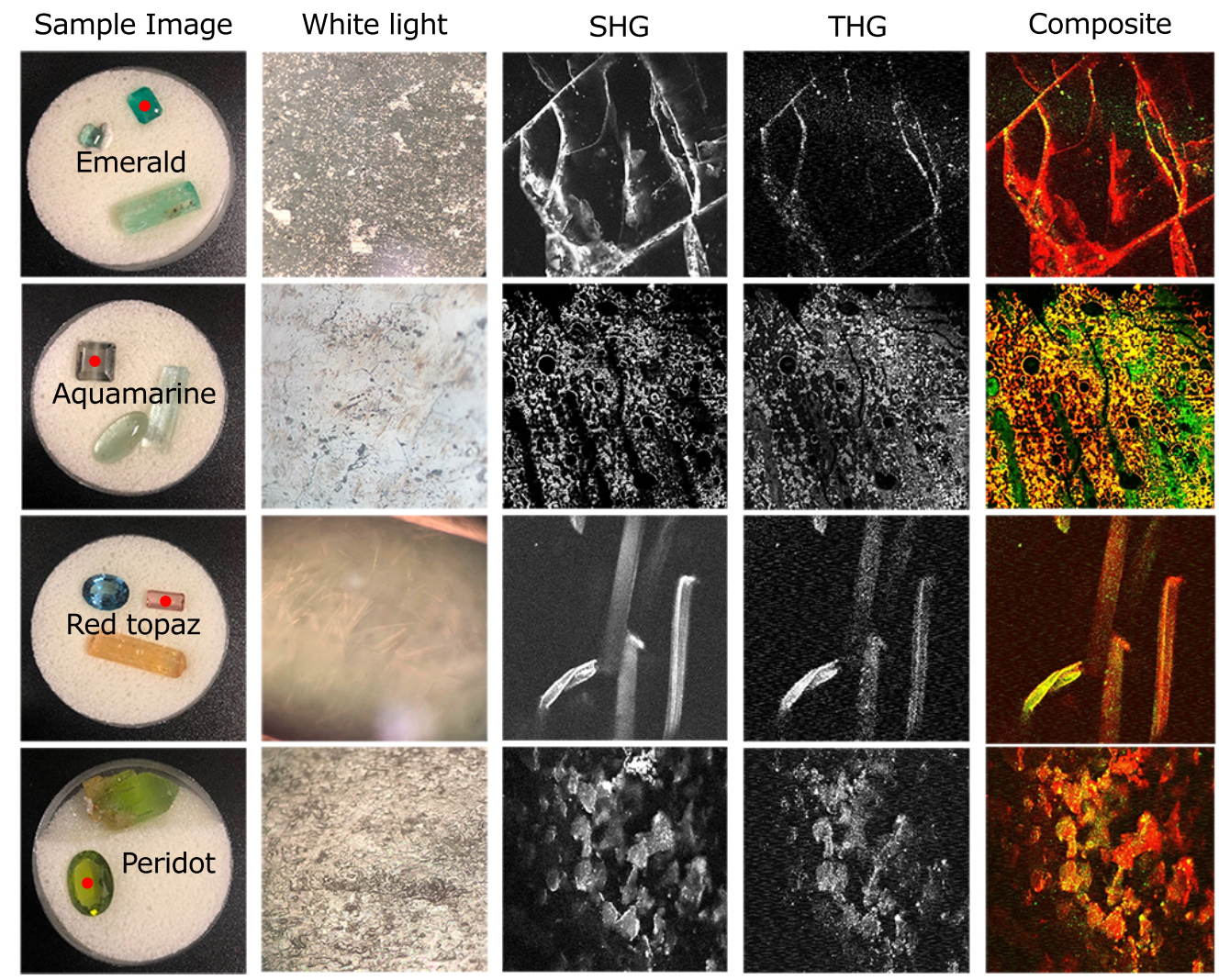

Figure 1. A collection of images of a subset of the birthstone samples. The left column shows labelled images of the samples themselves, with a red dot indicating which of the stones' images are displayed. The center left column shows the images under a white light microscope (Zeiss Acroplan 2). The center column has the SHG images from the samples. The center right column has the THG images. The rightmost column has a composite image, with SHG colored red, and THG colored green. Each multiphoton image is around $300 \mu \mathrm{m}$ across, and the white light images are around $500 \mu \mathrm{m}$ across

In Fig 1, images of the samples, and how they appear both to a white light microscope and a multiphoton microscope are displayed. The white light microscope can discern interesting features on the surface only for 
most of these samples. Details below the surface appear blurry primarily. The images from the multiphoton were obtained from a variety of depths in each sample. Monocrystalline samples tend to give less interesting information in 3D because of the fewer boundaries to give signal, but since SHG can be generated from bulk material, signal is still seen in non-centrosymmetric materials, such as minerals where the largest constituent is quartz. Quartz has a well-known and understood $\chi_{2}$ signal, ${ }^{23,24}$ leading to strong SHG in some samples. Several additional images of other birthstone samples are shown in Fig. 2
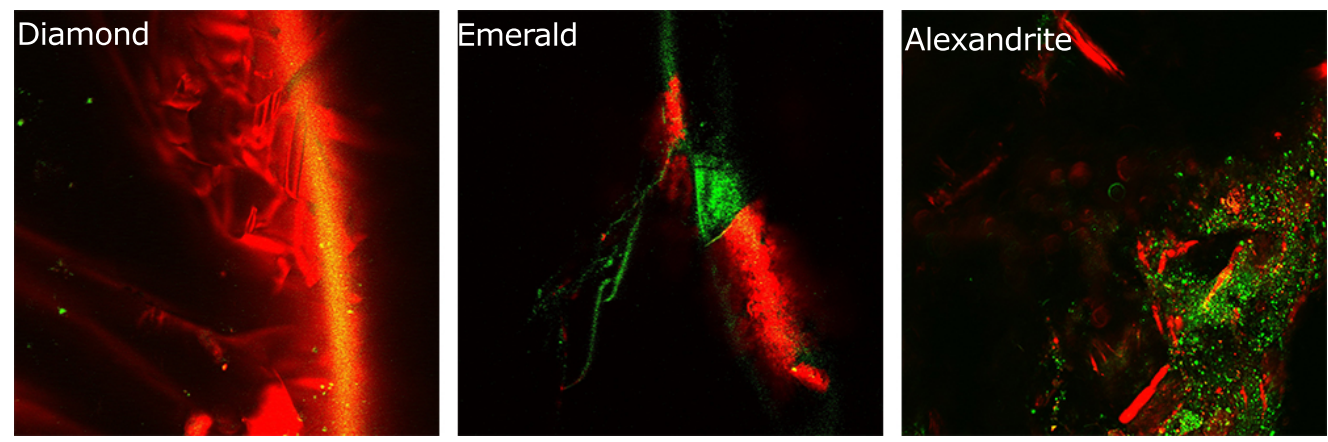

Figure 2. Composite multiphoton images of Diamond, Emerald, and Alexandrite. The image from the diamond had the most interesting structure near the edges as shown in this picture. As this stone was polished, perhaps there could be some structure changes after polishing that the multiphoton is highlighting

In our growing collection of minerals are several different types of Jasper, which is generally a microgranular or fibrous variety of quartz. Their commonly bright colors comes from various oxides within them. ${ }^{25}$ These stones produced some of the more visually striking images, and a collection of them can be seen in Fig. 3 below. The many small quartz crystals lead to both strong SHG from the crystalline structure of the quartz, and THG from the many boundaries, as well as any inclusions within the mineral.
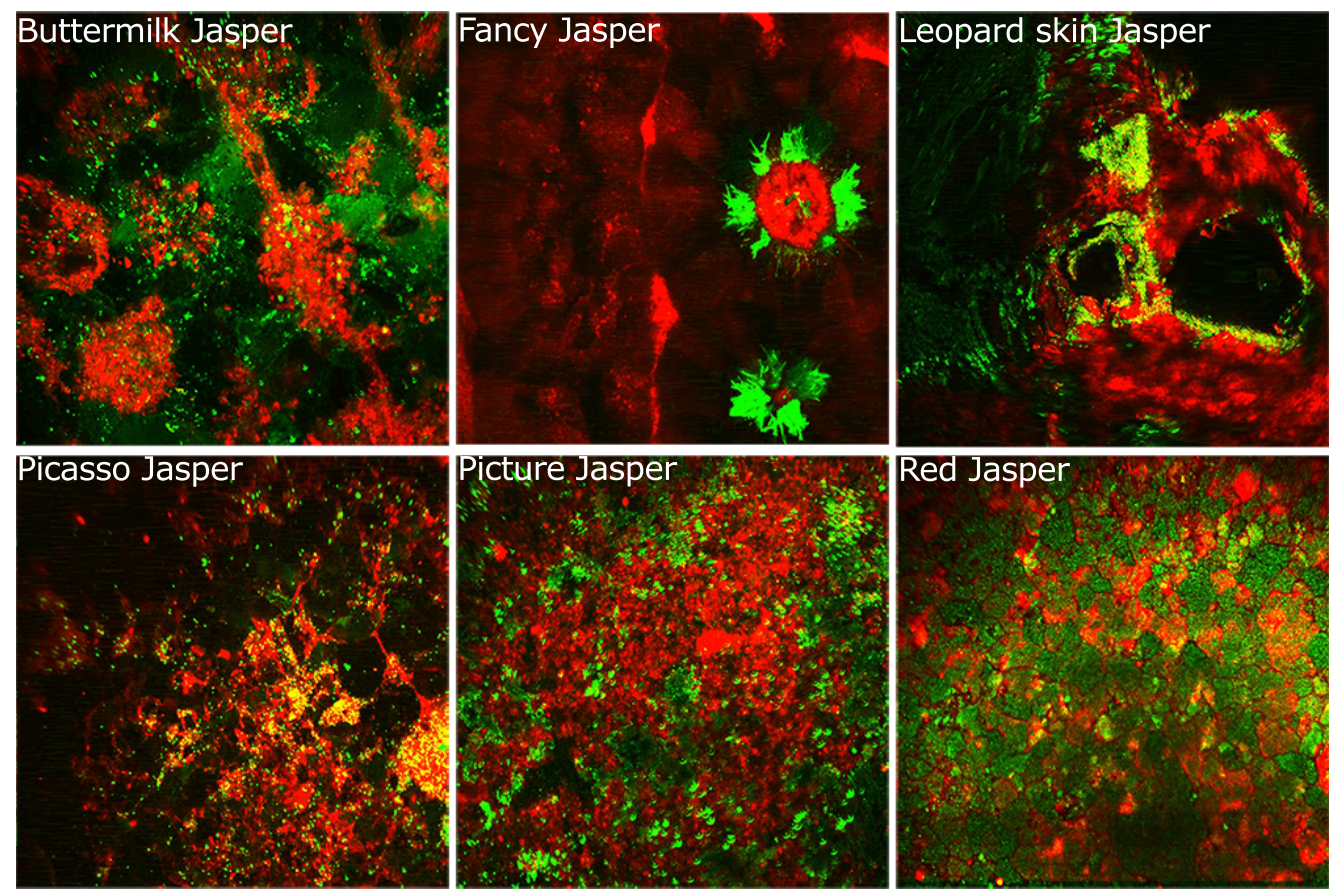

Figure 3. Our set of stones had several varieties of Jaspers, six of which are shown above in composite images from the multiphoton. Even within the same family of stones, the images can look quite different. The name of each type of Jasper is on each image. These images were captured using the $1040 \mathrm{~nm}$ laser.

One of the most interesting samples we examined was the Orange Calcite sample, a crystalline form of Calcium 
Carbonate. ${ }^{25}$ A variety of images from that sample are displayed in Fig. 4 below. This particular mineral was examined on the large and small scale, as well as in depth. On the large scale, individual grains or crystalline regions of the mineral can be seen. In this samples, the signal is primarily coming from the edges of each stone, such that as each grain goes through focus, the center is dark. As such, the dark regions in the image are not voids in the sample. While the SHG signal seemed similar to some of the other samples that were examined, the THG images had very finely structure variations, seen clearly in the THG image or the close up in Fig. 4 below. Capturing a large set of either depth or lateral images can be time consuming on our instrument, but this is not a limit of the technology, as very fast frame rate multiphoton microscopes have been reported in the literature. ${ }^{26}$ In our opinion, it is worth the wait to capture an image as fascinating as the stitched image of Fig. 4 . This stone also allowed for slightly deeper imaging than the average sample. In general, we could see 100-300 microns deep with the $1040 \mathrm{~nm}$ laser, and 300-600 microns deep with the 1550 laser. This sample allowed for imaging around 650 microns deep before the signal became weak enough to make getting good images more difficult.
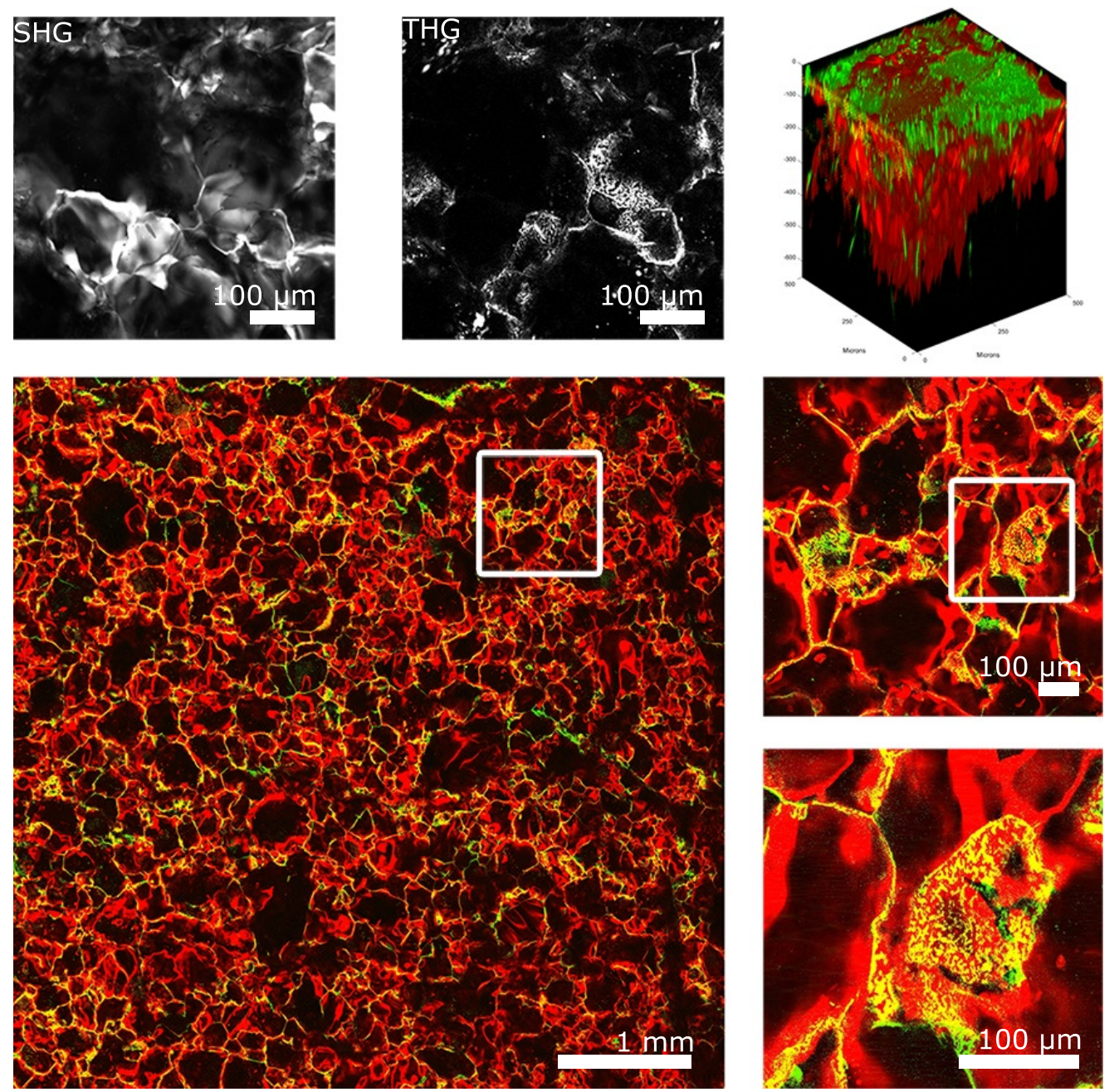

Figure 4. Top left and top center: an individual image taken around $100 \mu \mathrm{m}$ below the surface of an orange calcite sample. Top right: a compiled z stack of images. The zstack is around $400 \mu m$ deep and $500 \mu m$ on either edge. Bottom: A large stitched image with two insets progressively zooming in on the features of the stone. In the colored images, the SHG images are colored red, and the THG images are colored green. These images were captured with the $1550 \mathrm{~nm}$ laser.

As mentioned, the quartz-like samples often gave some of the strongest and most interesting images. Citrine, a birth stone for November, is a variety of quartz generally of yellow-orange color. This stone produced some very interesting triangular patterns in SHG, which could be indicative of Brazil pair twinning, a pattern that 
can occur between regions of quartz formation. ${ }^{27}$ These patterns are usually studied in thin sections under a polarized light microscope, which requires cutting and polishing the stone from a larger portion. It is usually difficult to place more than one twin boundary in a single thin section, ${ }^{27}$ so looking through multiple pairs in $3 \mathrm{D}$ is a useful new capability. A Zstack of the citrine sample can be seen in Fig. 5 below. A quartz sample was examined thoroughly in depth in our previous work, and the images there appear similar to the larger scale patterns seen in this sample. However, the triangular patterns seen in the highlighted depth of $z=67 \mu m$ to the right of the $3 \mathrm{D}$ rendering are a new observation.
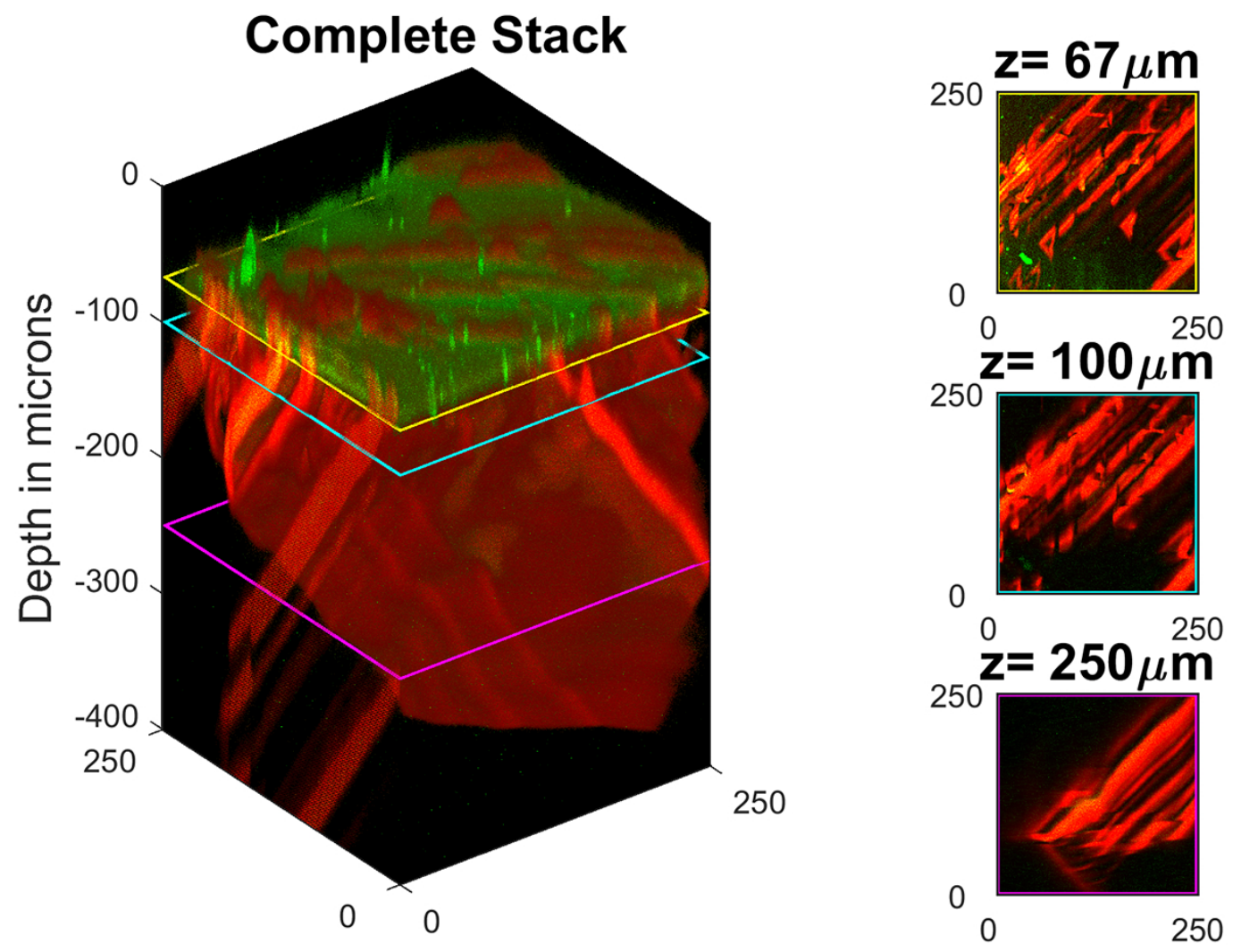

Figure 5. Left: A 3D rendering of a citrine sample. Clear triangluar patterns appear near the surface region of the stone, but wash out further down in the crystal. The deeper patterns appear similar to images we have taken previously of pure quartz samples, but the triangular patterns seen most clearly at $z=67 \mu \mathrm{m}$ are unique to this location in the Citrine.

The Tucson region is well known in the gem and mineral community due the large gem and mineral show that occurs every year, ${ }^{28}$ where our original sample set was purchased. In addition to this, the many mines in Arizona lead to a variety of locally found samples. Some of the minerals are very easy to access, and do not need to be mined by professionals. For example, it is common to see flakes of mica sparkling on the ground on a hike in any of the mountains surrounding Tucson. One such flake was brought back down to our microscope to be examined. Mica is a type of phyllosilicate, and is often made of many flakes that break apart from each other with little effort. ${ }^{25}$ Several of these flakes can be seen in Fig. 6 below, alongside highlighted depths. In this sample, different flake regions can be seen at different depths in the depth highlights to the right of the figure. The THG and SHG signals are generated by different portions of the sample. In the 3D view, the surface topology of the mica slices can be seen. 

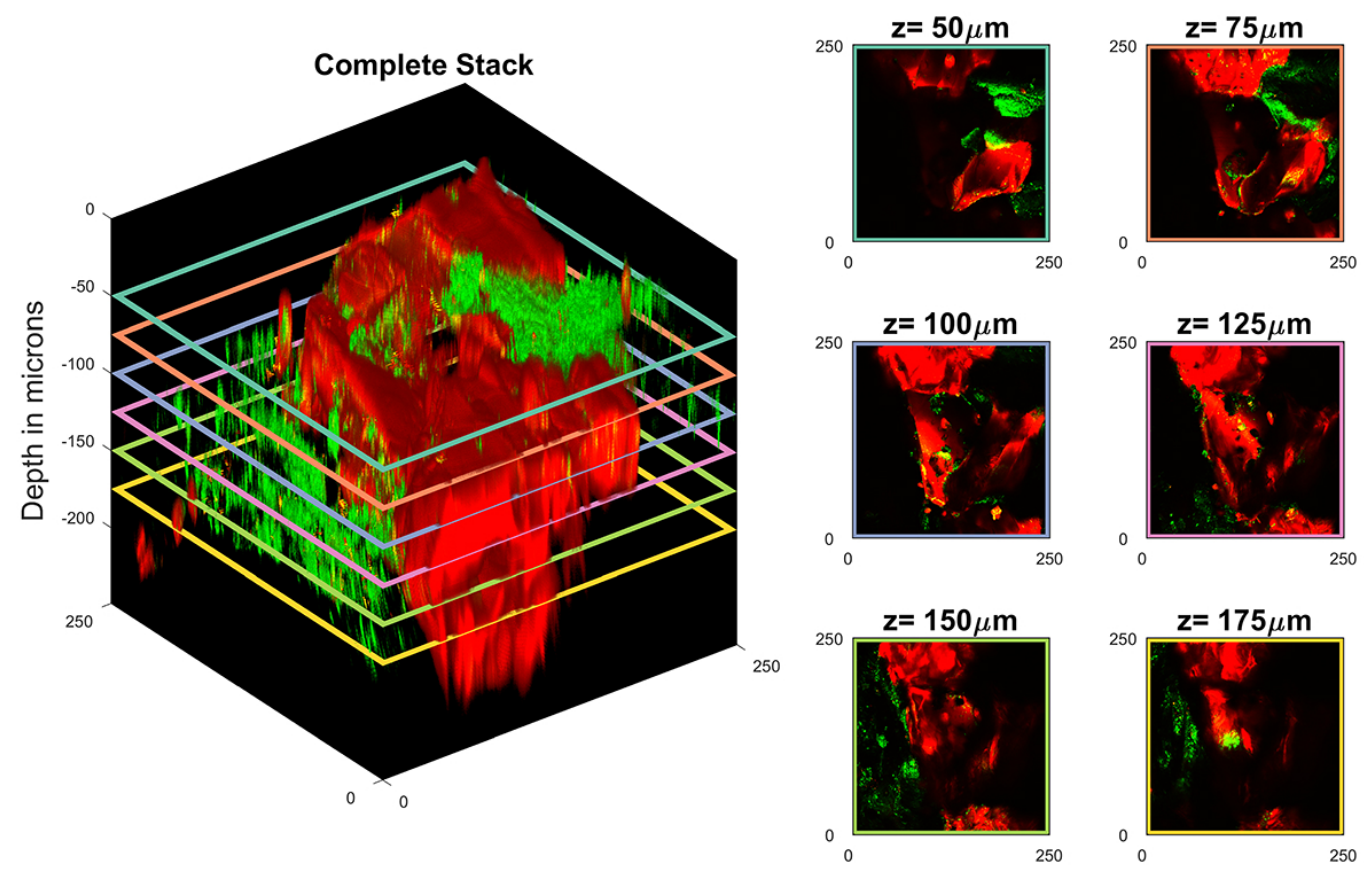

Figure 6. A Mica sample from Mt. Lemmon, which is just north of Tucson, AZ. In this Mica sample, the SHG and THG are clearly not being generated from the same structures within the mica sample. Individual flakes of the Mica can be seen in the different depths of the Zstack

\section{CONCLUSION}

We are very excited to continue to pursue this new application of multiphoton microscopy in the geological sciences. We are only beginning to understand the meaning of the information our instrument can capture within these precious stones. Understanding the images will require further work comparing our results to known approaches of examining samples in the gem and mineral community, which is a focus of upcoming work. There is much still to do in this brand new research direction, and we look forward to seeing what we can discover. The images we have captured in this project are some of the most beautiful pictures we have ever recorded on our instrument.

\section{ACKNOWLEDGMENTS}

The authors would like to thank Katarina Korch for her work performed during the Vail High School Internship program, in which she produced a few of the images in this paper. We would also like to offer our sincere gratitude to our new collaborators in the Geosciences department for lending us their collections of stones to study.

Funding for this work was provided by the National Science Foundation (NSF) through the Graduate Research Fellowship Program (GRFP) under DGE-1746060, and NSF ECCS 1610048.

\section{REFERENCES}

[1] Pirard, E., Lebichot, S., and Krier, W., "Particle texture analysis using polarized light imaging and grey level intercepts," International Journal of Mineral Processing 84(1), 299-309 (2007).

[2] Bersani, D. and Lottici, P. P., "Applications of raman spectroscopy to gemology," Analytical and Bioanalytical Chemistry 397(7), 2631-2646 (2010).

[3] Hope, G. A., Woods, R., and Munce, C. G., "Raman microprobe mineral identification," Minerals Engineering 14(12), 1565-1577 (2001). 
[4] Reiche, I., Pages-Camagna, S., and Lambacher, L., "In situ raman spectroscopic investigations of the adorning gemstones on the reliquary heinrich's cross from the treasury of basel cathedral," Journal of Raman Spectroscopy 35(8), 719-725 (2004).

[5] Barone, G., Bersani, D., Lottici, P. P., Mazzoleni, P., Raneri, S., and Longobardo, U., "Red gemstone characterization by micro-raman spectroscopy: the case of rubies and their imitations," Journal of Raman Spectroscopy 47(12), 1534-1539 (2016).

[6] Kiefert, L., Haenni, H. A., and Chalain, J.-P., "Identification of gemstone treatments with raman spectroscopy," in [Optical Devices and Diagnostics in Materials Science], 4098, 241-252, International Society for Optics and Photonics (2000).

[7] Giarola, M., Mariotto, G., Barberio, M., and Ajó, D., "Raman spectroscopy in gemmology as seen from a 'jeweller's' point of view," Journal of Raman Spectroscopy 43(11), 1828-1832 (2012).

[8] Culka, A., Hyršl, J., and Jehlička, J., "Gem and mineral identification using GL gem raman and comparison with other portable instruments," Applied Physics A 122(11), 959 (2016).

[9] Martin-Olmos, C., Stieg, A. Z., and Gimzewski, J. K., "Electrostatic force microscopy as a broadly applicable method for characterizing pyroelectric materials," Nanotechnology 23(23), 235701 (2012).

[10] Turner, D., Groat, L. A., Rivard, B., and Belley, P. M., "Reflectance spectroscopy and hyperspectral imaging of sapphire-bearing marble from the beluga occurrence, baffin island, nunavut," The Canadian Mineralogist 55(4), 787-797 (2017).

[11] Yu, Z., Cui, Y., Wang, X., and Zhang, Y., "Identification of ores and gems using THz polarization information," in [Infrared, Millimeter Wave, and Terahertz Technologies], 7854, 78541U, International Society for Optics and Photonics (2010).

[12] Han, D., Jeong, H., Song, Y., Ahn, J. S., and Ahn, J., "Lattice vibrations of natural seraphinite gemstone probed by terahertz time-domain spectroscopy," IEEE Transactions on Terahertz Science and Technology 5(6), 1021-1027 (2015).

[13] Krivanek, O. L., Chisholm, M. F., Nicolosi, V., Pennycook, T. J., Corbin, G. J., Dellby, N., Murfitt, M. F., Own, C. S., Szilagyi, Z. S., Oxley, M. P., Pantelides, S. T., and Pennycook, S. J., "Atom-by-atom structural and chemical analysis by annular dark-field electron microscopy," Nature 464(7288), 571-574 (2010).

[14] Sahoo, R. K., Singh, S. K., and Mishra, B. K., "Surface and bulk 3d analysis of natural and processed ruby using electron probe micro analyzer and x-ray micro CT scan," Journal of Electron Spectroscopy and Related Phenomena 211, 55-63 (2016).

[15] Cromey, B., Knox, R. J., and Kieu, K., "3d imaging of gems and minerals by multiphoton microscopy," Opt. Mater. Express 9, 516-525 (Feb 2019).

[16] Denk, W., Strickler, J., and Webb, W., "Two-photon laser scanning fluorescence microscopy," Science 248(4951), 73-76 (1990).

[17] Zipfel, W. R., Williams, R. M., and Webb, W. W., "Nonlinear magic: multiphoton microscopy in the biosciences," Nat Biotech 21, 1369-1377 (Nov 2003).

[18] Kieu, K., Mehravar, S., Gowda, R., Norwood, R. A., and Peyghambarian, N., "Label-free multi-photon imaging using a compact femtosecond fiber laser mode-locked by carbon nanotube saturable absorber," Biomed. Opt. Express 4, 2187-2195 (Oct 2013).

[19] Säynätjoki, A., Karvonen, L., Rostami, H., Autere, A., Mehravar, S., Lombardo, A., Norwood, R. A., Hasan, T., Peyghambarian, N., Lipsanen, H., Kieu, K., Ferrari, A. C., Polini, M., and Sun, Z., "Ultrastrong nonlinear optical processes and trigonal warping in MoS 2 layers," Nature Communications 8(1), 893 (2017).

[20] Säynätjoki, A., Karvonen, L., Riikonen, J., Kim, W., Mehravar, S., Norwood, R. A., Peyghambarian, N., Lipsanen, H., and Kieu, K., "Rapid large-area multiphoton microscopy for characterization of graphene," ACS Nano 7(10), 8441-8446 (2013).

[21] Karvonen, L., Säynätjoki, A., Huttunen, M. J., Autere, A., Amirsolaimani, B., Li, S., Norwood, R. A., Peyghambarian, N., Lipsanen, H., Eda, G., Kieu, K., and Sun, Z., "Rapid visualization of grain boundaries in monolayer mos2 by multiphoton microscopy," Nature Communications 8, 15714 EP - (Jun 2017). Article.

[22] Boyd, R. W., [Nonlinear Optics, Third Edition], Academic Press, Inc., Orlando, FL, USA, 3rd ed. (2008). 
[23] Shoji, I., Kondo, T., Kitamoto, A., Shirane, M., and Ito, R., "Absolute scale of second-order nonlinearoptical coefficients," Journal of the Optical Society of America B 14(9), 2268 (1997).

[24] Jerphagnon, J. and Kurtz, S. K., "Optical nonlinear susceptibilities: Accurate relative values for quartz, ammonium dihydrogen phosphate, and potassium dihydrogen phosphate," Physical Review B 1(4), 17391744 (1970).

[25] Dill, H. G., "The "chessboard" classification scheme of mineral deposits: Mineralogy and geology from aluminum to zirconium," Earth-Science Reviews 100(1), 1-420 (2010).

[26] Bewersdorf, J., Pick, R., and Hell, S. W., "Multifocal multiphoton microscopy," Opt. Lett. 23, 655-657 (May 1998).

[27] Spry, A., Turner, R. G., and Tobin, R. C., "Optical phenomena associated with brazil-twin boundaries in quartz," American Mineralogist 54(1), 117-133 (1969).

[28] Tucson Gem and Mineral Society, "Tucson Gem and Mineral Show." http://www.tgms.org/show/. 